\title{
Theoretical analysis on the applicability of traditional SPH method
}

\author{
ZHOU GuangZheng*, GE Wei \& LI JingHai \\ State Key Laboratory of Multiphase Complex Systems, Institute of Process Engineering, Chinese Academy of Sciences, Beijing 100190, China
}

Received January 25, 2013; accepted April 9, 2013; published online May 23, 2013

\begin{abstract}
As a fully Lagrangian, particle-based numerical method, the traditional smoothed particle hydrodynamics (SPH) generally suffers from the accuracy problem. To investigate the physical origins of this numerical error, the elastic effect between SPH particles is specifically identified by analogy with physical entities, and a unique non-dimensional number is proposed to evaluate the relative dominance of viscous to elastic effect. Through the simulation of two-dimensional Couette flow, the velocity profile and arrangement of particles are examined for various ratios of viscous to elastic effect. The effective viscosity of SPH particles decreases as this non-dimensional number increases, while the increase of particle number significantly reduces the effective viscosity only at lower ratio of viscous to elastic effect. The disparity among nominal viscous dissipation, total dissipation, and theoretical dissipation further confirms the presence of unphysical dissipation resulting from the elastic effect. In summary, due to the constraints from the Mach number and the ratio of viscous to elastic effect, there exists a critical Reynolds number below which the Newtonian behavior could be approximately obtained through suitable choice of model parameters.
\end{abstract}

smoothed particle hydrodynamics, fluid mechanics, accuracy, Couette flow, particle methods

Citation: Zhou G Z, Ge W, Li J H. Theoretical analysis on the applicability of traditional SPH method. Chin Sci Bull, 2013, 58: 2970-2978, doi: 10.1007/s11434013-5889-9

A better understanding of the flow mechanisms is usually of fundamental importance to various problems in science and engineering, where numerical simulation plays an increasingly significant role. As a fully meshless particle method, smoothed particle hydrodynamics (SPH) is particularly advantageous for flows with complex and deforming boundaries. Resembling other particle methods, the continuous media in SPH are represented by a collection of numerical particles with associated physical properties. And the SPH discretization scheme reduces the general conservation laws of continuum fluid dynamics into a set of ordinary differential equations reminiscent of conventional molecular dynamics (MD). Those particles interact with their neighboring particles based on these equations, and their locations are further updated at each time step. In contrast to varieties of complicated interface tracking and/or capturing techniques in the grid-based methods, such as level set method, marker and cell (MAC), and volume of fluid (VOF), the

*Corresponding author (email: gzzhou@ @ome.ipe.ac.cn) interfaces in SPH are naturally identified according to the distribution of particles in a straightforward manner, regardless of the number of phases involved. Originally developed for astrophysical applications [1,2], SPH has been extended to a wide range of problems in fluid dynamics and even solid mechanics, including free surface flows $[3,4]$, multi-phase flows [5,6], non-Newtonian flows [7,8], turbulent flows [9,10], and fluid-structure interaction [11].

Nevertheless, the traditional SPH generally suffers from several drawbacks, including low accuracy, tensile instability, and difficulty in enforcing essential boundary condition [12]. In regard to the analysis of numerical accuracy, the consistency (or completeness) problem in SPH has been widely investigated $[13,14]$. In nature, due to the adaptive character of SPH method, the disordered arrangement of particles leads to the inconsistency in the approximation of function and its derivative operators [13,15]. To remedy these drawbacks of the conventional SPH, various methods have been developed accordingly. In the so-called reproducing kernel particle method [16], the consistency of SPH 
is improved through the construction of correction function. Based on Taylor series expansion, corrective smoothed particle hydrodynamics method (CSPM) [17] and finite particle method (FPM) [18] are developed for non-linear dynamic problems and viscous flows, respectively. Jiang et al. [19] further proposed the mixed corrected symmetric SPH (MCSSPH), which show higher accuracy and better stability in various problems. Moreover, the moving-least-squares-particle hydrodynamics (MLSPH) [20] was derived by means of Galerkin approximation and new particle volume which ensures thermodynamic compatibility. To enhance the accuracy of density and pressure fields, Chaniotis et al. [21] further employed the remeshing procedure through the periodic reinitialization of particle locations, and thus artificially restores the regularity of particle distribution.

Generally, most modifications of classical SPH are numerically complicated, and also demand quite large computational effort. Besides, some revised versions of SPH even lose the attractive meshless character of particle methods, resulting from the introduction of background meshes. Due to its conceptual simplicity and ease of implementation, the traditional SPH is the most extensively applied method. On the other hand, Monaghan [22] have mentioned that the particle methods are inherently linked to the underlying physics of fluid at micro-scale and/or meso-scale in some sense. Actually, concerning the SPH formulations of inviscid flow of adiabatic ideal gas, their similarities to MD method have been intensively explored in many aspects $[23,24]$. The SPH approach even has been extended to the so-called smoothed dissipative particle dynamics (SDPD) [25] for mesoscopic problems, where thermal fluctuations are incorporated in a physically consistent way. Besides, based on the physical origins of surface tension, surface tension of immiscible fluids has been successfully reproduced in SPH simulations simply by introducing attractive forces among all particles [26,27] or suitable repulsive forces only between particles of different phases [5,28,29]. These complicated phenomena all indicate certain isomorphism between SPH and the underlying physical particles on the micro-scale, and thus the simple form of SPH formulations is far beyond a mathematical description of the macroscopic flow.

Besides, although there exist certain guides for the accurate simulation of incompressible flow, the selection of model parameter is somewhat arbitrary in theory. Note that the non-dimensional numbers generally play an important role in the identification of different flow patterns under various circumstances, such as the Reynolds number, Froude number, and Weber number. Therefore, it is of great significance to understand the physical mechanism and applicability of traditional SPH from the viewpoint of its inherent non-dimensional number. Based on the previous work [30], a unique non-dimensional number is further proposed to characterize the relative dominance of viscous to elastic effect in this study. The influence of this non-dimensional number on the physical behavior of SPH particles is intensively investigated, while the findings indicate that this nondimensional number could serve as a criterion to evaluate the deviation of SPH simulations from Newtonian fluid.

\section{Numerical model}

\subsection{Basic SPH formulations}

The SPH method is essentially based on the interpolation theory. Specifically, an arbitrary function $f(\boldsymbol{r})$ at position $\boldsymbol{r}$ could be evaluated as sums over its neighboring positions with the following discrete form

$$
f(\boldsymbol{r})=\sum_{j} \frac{m_{j}}{\rho_{j}} f\left(\boldsymbol{r}_{j}\right) W\left(\boldsymbol{r}-\boldsymbol{r}_{j}, h\right),
$$

where $m_{j}$ and $\rho_{j}$ are the mass and density of particle $j$ located at position $\boldsymbol{r}_{j}$, while $W\left(\boldsymbol{r}-\boldsymbol{r}_{j}, h\right)$ is the weighting function with smoothing length $h$. Similarly, the gradient $\nabla f(\boldsymbol{r})$ could be calculated by

$$
\nabla f(\boldsymbol{r})=\sum_{j} \frac{m_{j}}{\rho_{j}} f\left(\boldsymbol{r}_{j}\right) \nabla W\left(\boldsymbol{r}-\boldsymbol{r}_{j}, h\right) .
$$

The weighting function should tend to Dirac delta function as the smoothing length approaches zero, and also satisfy the normalization condition

$$
\int W\left(\boldsymbol{r}-\boldsymbol{r}^{\prime}, h\right) \mathrm{d} \boldsymbol{r}^{\prime}=1 .
$$

Since the second derivative of weighting function is critical to the stability of SPH [31], the two-dimensional version of quintic spline is adopted in this work, due to its continuous second derivative.

Following eq. (1), the SPH formulation of local density is

$$
\rho_{i}=\sum_{j} m_{j} W\left(\left|\boldsymbol{r}_{i}-\boldsymbol{r}_{j}\right|, h\right),
$$

which exactly satisfies the mass conservation in theory. Based on the symmetric form of pressure gradient which preserves variational consistency and the viscous diffusion term evaluated by means of integral approximation, a popular SPH formulation of Navier-Stokes equation [26,31] is written as

$$
\begin{aligned}
\frac{\mathrm{d} \boldsymbol{v}_{i}}{\mathrm{~d} t}= & -\sum_{j} m_{j}\left(\frac{P_{i}}{\rho_{i}^{2}}+\frac{P_{j}}{\rho_{j}^{2}}\right) \nabla_{i} W_{i j} \\
& +\sum_{j} \frac{m_{j}\left(\mu_{i}+\mu_{j}\right)\left(\boldsymbol{v}_{i}-\boldsymbol{v}_{j}\right)}{\rho_{i} \rho_{j}}\left(\frac{1}{r_{i j}} \frac{\partial W_{i j}}{\partial r_{i}}\right)+\boldsymbol{g},
\end{aligned}
$$

where $P_{i}, \mu_{i}$, and $\boldsymbol{v}_{i}$ are the pressure, dynamic viscosity, and velocity of particle $i$, respectively. Also, $r_{i j}$ is the scale distance between particles $i$ and $j, W_{i j}=W\left(\boldsymbol{r}_{i}-\boldsymbol{r}_{j}, h\right)$, and $\nabla_{i}$ 
denotes the gradient with respect to the coordinates of particle $i$. The index $i$ of eq. (5) runs over all fluid particles, while the index $j$ runs over all adjacent particles of particle $i$, which include both fluid particles and boundary particles.

In regard to the incompressibility constraint, the employment of actual equation of state requires a prohibitively small time step. Instead, the incompressible flow is generally approximated through artificial equation of state. A simple and typical state equation $[27,31,32]$ has the form

$$
P=c^{2} \rho=K \rho,
$$

where $c$ is the numerical sound speed, and $K$ is termed as "stiffness parameter". In practice, large numerical sound speed is usually required to guarantee sufficiently small density fluctuation. According to Monaghan [3], the relation between density fluctuation $\delta$ and Mach number Ma would be

$$
\delta=\frac{\Delta \rho}{\rho_{0}} \approx \mathrm{Ma}^{2}=\left(\frac{V_{\max }}{c}\right)^{2}=\frac{V_{\max }^{2}}{K},
$$

where $V_{\max }$ is the maximum velocity. As argued by Morris et al. [31], the largest density fluctuation should be no more than $3 \%$ (typically set to be $1 \%$ ) to ensure relatively high accuracy in the pressure field.

\subsection{Boundary conditions}

Compared with grid-based numerical methods, the treatment of rigid boundary in SPH is much more complex. Generally, there exist three major strategies, using repulsive force, mirror particles, and boundary particles, respectively. The repulsive force method proposed by Monaghan $[3,22]$ introduces a layer of particles directly deployed on the surface of boundary, which exerts strong repulsive force on fluid particles approaching the boundary. Although this technique effectively prevents the unphysical penetration of fluid into the rigid boundary, the fluid particles adjacent to the boundary would have incomplete support of neighboring particles. Another typical approach utilizes mirror particles $[33,34]$ through reflecting the inner fluid particles across the rigid boundary, but this technique suffers from the difficulty in handling complex geometries. As for the third method of boundary treatment, several layers of fixed boundary particles are directly extended from the initial regular distribution of inner fluid particles $[31,35,36]$. These boundary particles contribute to the relevant evaluations of surrounding fluid particles, and are computationally more efficient compared with mirror particles.

In regard to the implementation of non-slip boundary condition in SPH, fictitious velocities are generally associated with boundary particles based on the reasonable assumption of a linear velocity distribution near the boundary $[31,35]$. More specifically, as sketched in Figure 1, when a fluid particle $i$ of velocity $\boldsymbol{v}_{i}$ interacts with a boundary particle

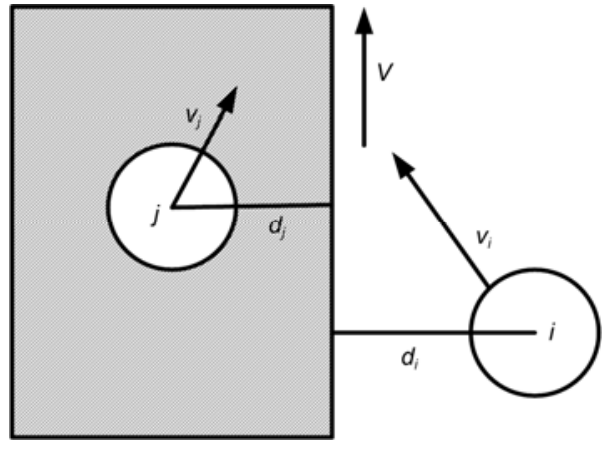

Figure 1 Schematic of no-slip boundary condition.

$j$, the fictitious velocity of particle $j$ would be

$$
\boldsymbol{v}_{j}=\boldsymbol{V}+\frac{d_{j}}{d_{i}}\left(\boldsymbol{V}-\boldsymbol{v}_{i}\right),
$$

where $d_{i}$ and $d_{j}$ are the normal distances from particles $i$ and $j$ to the boundary, respectively, while $\boldsymbol{V}$ is the driving velocity of the rigid boundary. If the boundary is stationary, eq. (8) further simplifies to

$$
\boldsymbol{v}_{j}=-\frac{d_{j}}{d_{i}} \boldsymbol{v}_{i} .
$$

Moreover, an artificial Neumann pressure condition is commonly enforced on rigid boundary $[10,34]$.

\subsection{Ratio of viscous to elastic effect}

When the pressure and dynamic viscosity of particles in eq. (5) is replaced by the state equation (eq. (6)) and fluid viscosity $\left(\mu_{0}\right)$, respectively, and the gravity term is neglected, eq. (5) further reduces to

$$
\begin{aligned}
\frac{\mathrm{d} \boldsymbol{v}_{i}}{\mathrm{~d} t}= & -K \sum_{j} m_{j}\left(\frac{1}{\rho_{i}}+\frac{1}{\rho_{j}}\right) \nabla_{i} W_{i j} \\
& +2 \mu_{0} \sum_{j} \frac{m_{j}\left(\boldsymbol{v}_{i}-\boldsymbol{v}_{j}\right)}{\rho_{i} \rho_{j}}\left(\frac{1}{r_{i j}} \frac{\partial W_{i j}}{\partial r_{i}}\right) .
\end{aligned}
$$

As for the first term of the right-hand side of eq. (10) (the pressure term), the local densities of particles $i$ and $j$ are solely determined by their neighboring particle distribution, while the gradient of the weighting function depends on the relative locations of these two particles. Therefore, with a given particle distribution, the stiffness parameter $K$ completely determines the value of pressure term. In fact, the interparticle force corresponding to the pressure term physically resembles the normal force of elastic spheres in some aspects. When SPH particles partially overlap with each other, their densities rapidly increase, resulting in large repulsive force between them in the normal direction. In regard to the physical entities of elastic sphere, the increment of elastic force is indeed proportional to the local strain of 
particles in certain way, such as the physically reasonable Hertz model and the simplified linear spring model widely applied in the discrete element method (DEM) of granular materials [37,38]. The stiffness parameter of $\mathrm{SPH}$, the Young module of Hertz model, and the interparticle stiffness of DEM essentially characterize the strength of repulsive force between particles in similar manners. Therefore, the influence of stiffness parameter on flow behavior is particularly termed as "elastic effect".

On the other hand, it is revealed by the last term of eq. (10) that the viscous effect of SPH particles is directly proportional to the fluid viscosity. The viscous (dissipative) and elastic (approximately conservative) effects strongly couple with each other among SPH particles in our system. Actually, the dissipative (irreversible) and conservative (reversible) processes are quite common in various non-equilibrium systems, for which a general equation of the non-equilibrium reversible-irreversible coupling has be proposed [39]. Recalling the formulation of viscous stress of incompressible fluid, the relative dominance of viscous to elastic effect in SPH could be described by the following non-dimensional number

$$
\frac{v_{0} \dot{\gamma}}{K}=\frac{v_{0} \rho \dot{\gamma}}{K \rho}=\frac{\mu_{0} \dot{\gamma}}{K \rho}=\frac{\text { Viscous effect }}{\text { Elatic effect }},
$$

where $v_{0}$ is the nominal kinematic viscosity, and $\dot{\gamma}$ is the shear rate. This non-dimensional number would be referred to as "ratio of viscous to elastic effect" hereafter, while its implications on the physical behavior of SPH particles are explored as follows.

\section{Results and discussion}

The simulation of two-dimensional plane Couette flow (in the vertical direction) is carried out with identical computational domain of our previous work [30]. In this benchmark problem, viscous fluid is sheared between two parallel and infinite plates, one of which is moving relative to the other. As illustrated in Figure 2, the boundaries are constructed through the extension of fluid domain for a cut-off distance of the weighting function ( $3 h$ for quintic spline). The overall computational domain is initially established by uniform distribution of particles (with equal constant masses) in a densely packed array of hexagonal lattice, while those ones located in the interior of boundaries are specified as boundary particles. The horizontal and vertical directions are specified as the $x$ and $y$ directions, respectively. Periodic boundary conditions are applied in the $y$ direction, so that the particles adjacent to the two involved boundaries interact with each other due to the periodical replication of the simulated region. In order to study a uniform shear flow, gravity is neglected in the present work.

In our cases, the distance between the two boundaries is $1.0 \times 10^{-3} \mathrm{~m}$, the density of the fluid is $1.0 \times 10^{3} \mathrm{~kg} \mathrm{~m}^{-3}$, and

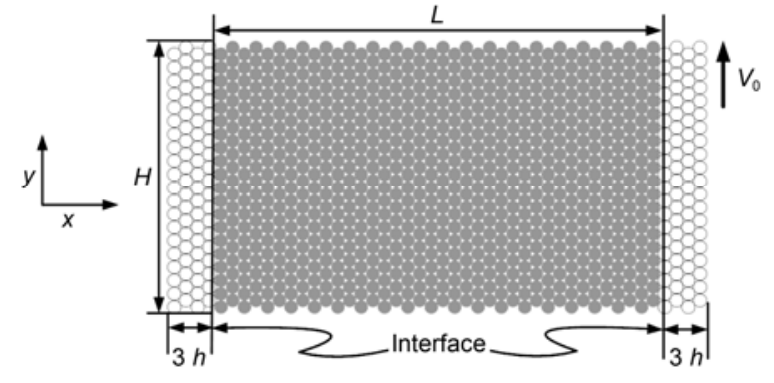

Figure 2 Schematic of SPH simulation of Couette flow. Fluid particles and boundary particles are represented by solid and hollow circles, respectively. The right boundary moves upwards with a constant velocity $V_{0}$. Adapted from Ref. [30].

the nominal kinematic viscosity is $2.0 \times 10^{-6} \mathrm{~m}^{2} \mathrm{~s}^{-1}$. For convenience, reduced units are employed in the simulations hereafter, where the cut-off distance of the weighting function $\left(1.0 \times 10^{-4} \mathrm{~m}\right)$, the mass of each particle $\left(7.826 \times 10^{-11} \mathrm{~kg}\right)$, and the kinematic viscosity are all equal to unity, resulting in a time unit of $5.0 \times 10^{-3} \mathrm{~s}$. Hence, both density $\rho_{0}$ and nominal dynamic viscosity $\mu_{0}$ of the fluid are 12.78 , while the distance between the two boundaries is $L=10.0$, and their lengths are $H=6.0$. The initial particle spacing is 0.3 , and the explicit Euler scheme is employed to perform the time integration with a time step of 0.02 .

\subsection{Velocity deviation}

With the driving velocity of right boundary $V_{0}=0.2$ and $K=$ 1.625 (corresponding to $v_{0} \dot{\gamma} / K=1.231 \times 10^{-2}$ ), Figure 3 presents the comparison of tangential velocity $\left(\boldsymbol{v}_{y}\right)$ profiles at several instants between SPH simulation and the series solution of its Newtonian fluid counterpart [31]. Note that the velocity fields of series solution and SPH results display nearly identical evolution process, both of which eventually become linear at the steady state. The numerical error of SPH simulation is only $0.035 \%$ in terms of the velocity of the rightmost particle, confirming the accurate prediction of the Newtonian behavior in this case. Additionally, the tangential force (along the $y$ direction) felt by a rightmost fluid particle from the boundary quickly decreases from initial 3.4725 to 0.0604 almost within a time of 50, and the fluctuation amplitude in the steady state is only $1.455 \times 10^{-3}$. Meanwhile, the perpendicular force (along the $x$ direction) from the boundary displays periodic oscillations around 4.905 with an amplitude of $1.969 \times 10^{-3}$, accounting for only about $3 \%$ of the tangential force. In summary, the no-slip boundary condition yields much more reliable results as compared with the method of boundary force [3] which tends to result in large perturbations of pressure field. To avoid dramatic acceleration of SPH particles, the driving velocity would linearly increase from zero to the final value within a time of $1.0 \times 10^{4}$ hereafter.

To investigate the influence of the ratio of viscous to 


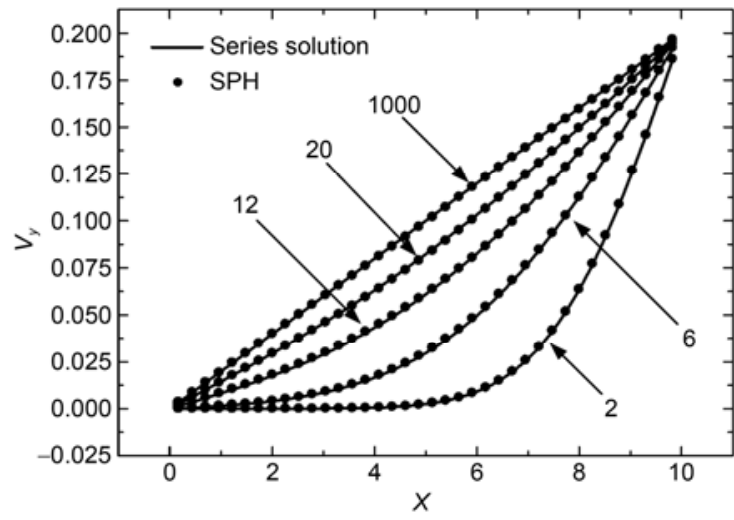

Figure 3 Comparison of tangential velocity profiles between SPH simulation and series solution at several instants.

elastic effect on the dynamics of SPH particles, several cases with different driving velocities are examined under the stiffness parameter $K=6.5$. It is found that the steady-state velocity profile oscillates around the linear distribution. The amplitude of oscillation gradually decreases as $v_{0} \dot{\gamma} / K$ increases, and closely approximates zero when $v_{0} \dot{\gamma} / K$ is sufficiently large. Based on our previous work [30], Figure 4 further presents the typical snapshots of simulation results with $V_{0}=0.0,2.5 \times 10^{-2}$, and 0.4 , corresponding to $v_{0} \dot{\gamma} / K=$
0.0 (a), $3.846 \times 10^{-4}$ (b), and $6.154 \times 10^{-3}$ (c), respectively. Notice that the velocity profile and arrangement of particles gradually vary with the increase of $v_{0} \dot{\gamma} / K$, ranging from the state completely dominated by elastic effect where all particles move at the same rate (stationary in limit) with uniform arrangement (Figure 4(a)), to the other state dominated by viscous effect where there is purely linear velocity profile accompanied by almost disordered structure of particle distribution (Figure 4(c)). As for the intermediate state, Figure 4(d) displays a closer view of the boxed region in Figure 4(b), where the velocity profile considerably deviates from its theoretical counterpart. Besides, particles arrange in form of hexagonal lattice in the plateau sections of velocity profile, whereas they arrange in form of square lattice in the slope sections. Actually, since the incompressibility is approximately ensured in $\mathrm{SPH}$, the viscous term of momentum equation (eq. (10)) is basically determined by the velocity profile. Meanwhile, as elucidated in Section 1.3, the elastic effect completely depends on the particle arrangement under a given stiffness parameter. Therefore, the balance between elastic and viscous effects accounts for the particular correspondence between velocity profile and particle arrangement at the steady state.

In view of the different flow patterns of SPH simulation, the physical behavior of classical SPH is basically parameter-

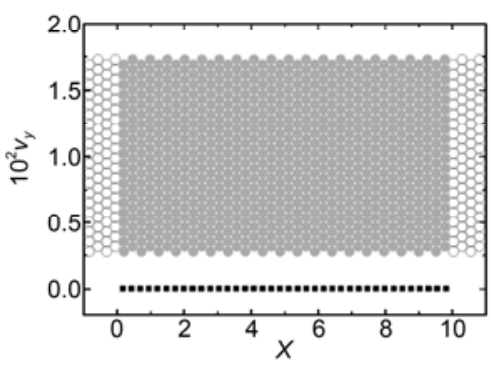

(a)

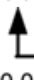

Elastic effect dominated
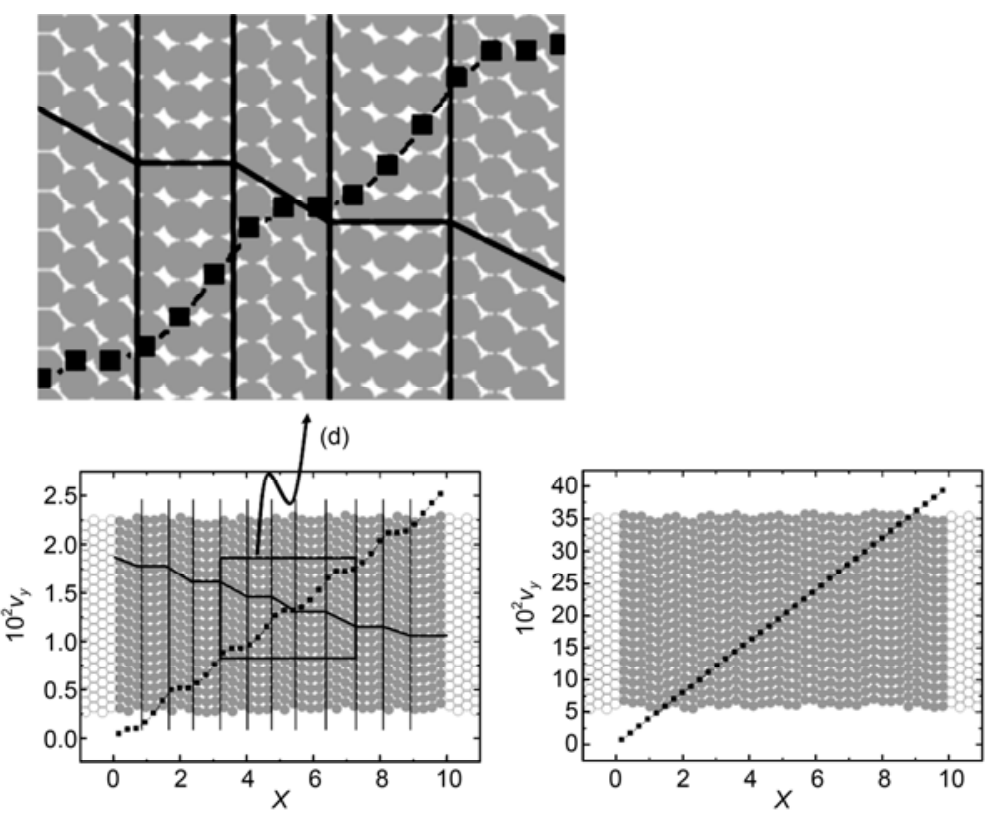

(b)

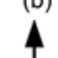

$3.846 \times 10^{-4}$

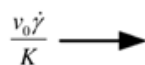

Viscous effect dominated

Figure 4 Dependence of velocity profile and particle arrangement on the non-dimensional number. (a) $v_{0} \dot{\gamma} / K=0$; (b) $3.846 \times 10^{-4}$; (c) $6.154 \times 10^{-3}$; (d) a closer view of the boxed region in (b). The vertical lines in (b) separate different layers, while the slope and plateau sections of solid lines denote local particle arrangement in hexagonal and square patterns, respectively. Adapted from Ref. [30]. 
dependent. The purely Newtonian fluid could be well approximated only under sufficiently large $v_{0} \dot{\gamma} / K$, and thus this non-dimensional number could serve as a criterion to evaluate the deviation of SPH simulation from Newtonian fluid. Besides, the ratio of viscous to elastic effect can be directly linked to the Mach number with the following form

$$
\frac{v_{0} \dot{\gamma}}{K}=\frac{v_{0}}{K}\left(\frac{V_{\max }}{L}\right)=\frac{v_{0}}{L K}\left(\mathrm{Ma}^{0.5}\right)=\frac{v_{0}}{L K^{0.5}} \mathrm{Ma} .
$$

As indicated by eq. (12), the available values of $v_{0} \dot{\gamma} / K$ with a given stiffness parameter is restricted to a certain range due to the incompressibility constraint on Mach number.

\subsection{Effective viscosity}

In regard to the effective viscosity of SPH particles, the influence of the ratio of viscous to elastic effect is also investigated under a wide range of stiffness parameter and shear rate. With the measurement of tangential stress $\tau$ between fluid particles and the right moving boundary, the effective viscosity $\mu$ can be simply calculated by $\tau / \dot{\gamma}$. Actually, the tangential stress at the steady state also oscillates around a certain value, so reliable time-averaged values are obtained with sufficiently long time. Figure 5 presents the relationship between effective viscosity and the ratio of viscous to elastic effect. It is found that those curves of different stiffness parameters closely match each other, indicating the dependence of effective viscosity on the nondimensional number $v_{0} \dot{\gamma} / K$, rather than stiffness parameter or shear rate. Notice also that the effective viscosity quickly decreases as the ratio of viscous to elastic effect increases, and it closely approximates the nominal viscosity when $v_{0} \dot{\gamma} / K$ is sufficiently large. Accordingly, to accurately predict the flow behavior of Newtonian fluid, it is quite important to ensure sufficiently large ratio of viscous

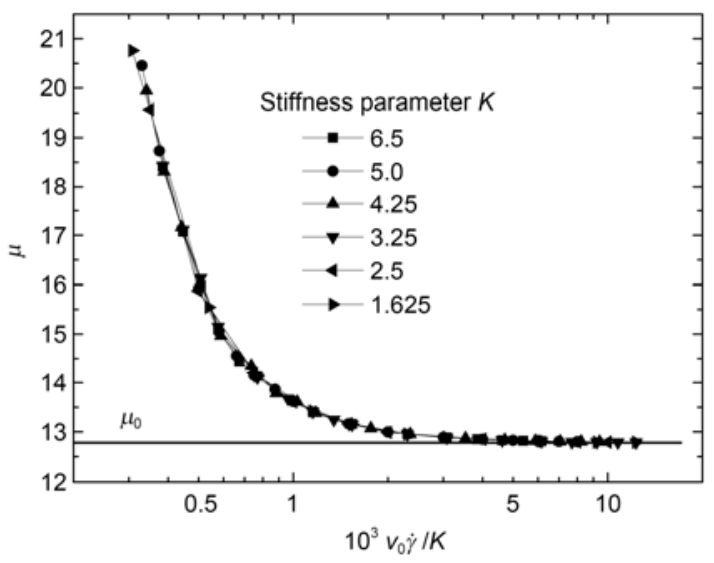

Figure 5 Variations of effective viscosity with the ratio of viscous to elastic effect under different stiffness parameters. to elastic effect in SPH simulations.

On the other hand, it is generally believed that the SPH method would have reasonable accuracy, when sufficiently large number of fluid particles is involved in the simulation. Thus, the influence of particle number on effective viscosity is further investigated for various ratios of viscous to elastic effect (changing shear rate with $K=6.5$ ). It should be mentioned that, the initial particle spacing, smoothing length, and mass of each particle all decrease with the increase of particle number, but the computational results herein have been transformed with the same reduced units as that of previous cases with particle number $n=760$. As illustrated in Figure 6 , the effective viscosity of several particle numbers (including 760, 1710, 3080, and 4800) all decreases with increasing $v_{0} \dot{\gamma} / K$, and closely approximates the nominal viscosity when $v_{0} \dot{\gamma} / K$ is sufficiently large. It is also noted that as particle number increases, the effective viscosity significantly decreases at relatively lower $v_{0} \dot{\gamma} / K$. Nevertheless, the disparity among the effective viscosity of these particles is not remarkable when $v_{0} \dot{\gamma} / K$ is larger than certain value (such as $7.692 \times 10^{-4}$ ). In summary, when $v_{0} \dot{\gamma} / K$ is sufficiently large, the result with relatively few particles $(n=760)$ would be quite acceptable, while further increase of particle number cannot significantly improve the accuracy. Actually, as revealed in Figure 6, the effective viscosity of sufficiently large $v_{0} \dot{\gamma} / K$ even somewhat deviates from nominal viscosity for the particle numbers 3080 and 4800 , due to certain numerical errors.

To guarantee sufficiently large ratio of viscous to elastic effect, the corresponding stiffness parameter should be small enough for a given shear rate. However, the stiffness parameter should also be large enough to satisfy incompressibility constraint. Consequently, suitable choice of stiffness parameter would be a compromise of these two competing factors. In view of the maximum density fluctuations $\delta=0.03$ [31], the selection of stiffness parameter around

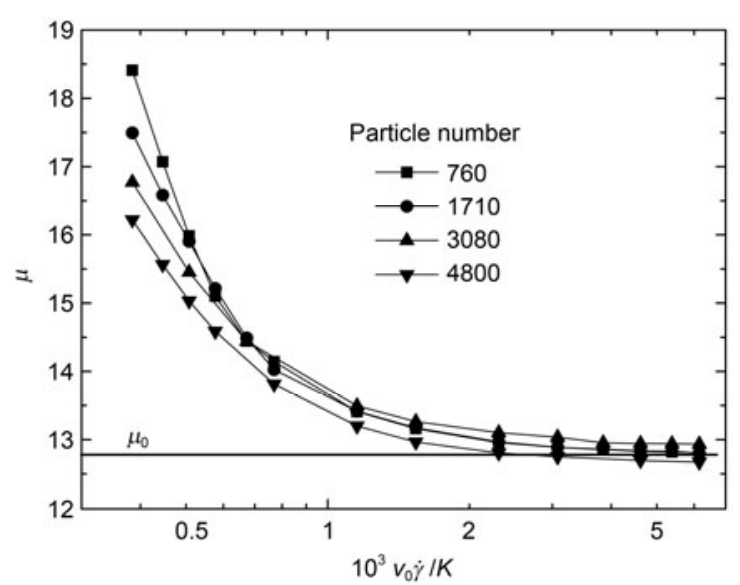

Figure 6 Variations of effective viscosity with the ratio of viscous to elastic effect under various numbers of fluid particle. 
$30 V_{\max }^{2}$ would be reasonable to some extent. On the other hand, when $K$ and $\dot{\gamma}$ are substituted with eq. (7) and $V_{\text {max }} / L$, respectively, the ratio of viscous to elastic ratio can be further rewritten as

$$
\frac{v_{0} \dot{\gamma}}{K}=v_{0}\left(\frac{\mathrm{Ma}}{V_{\text {max }}}\right)^{2}\left(\frac{V_{\text {max }}}{L}\right)=\frac{v_{0}}{V_{\text {max }} L} \mathrm{Ma}^{2}=\frac{\mathrm{Ma}^{2}}{\mathrm{Re}} .
$$

As indicated by eq. (13), with a given Mach number, the value of $v_{0} \dot{\gamma} / K$ is inversely proportional to the Reynolds number. Therefore, the traditional SPH method is actually more suitable for the simulations of low Reynolds number flows, which correspond to comparatively larger $v_{0} \dot{\gamma} / K$, and thus would have relatively higher accuracy. Besides, according to the variations of velocity profile (Figure 4) and effective viscosity (Figures 5 and 6), the Newtonian behavior can be approximately obtained when the non-dimensional number $v_{0} \dot{\gamma} / K$ is larger than $3 \times 10^{-3}$. Accordingly, there exists a critical Reynolds number, which could be evaluated by

$$
\mathrm{Re}=\frac{\mathrm{Ma}^{2}}{v_{0} \dot{\gamma} / K}<\frac{\mathrm{Ma}^{2}}{3 \times 10^{-3}}<\frac{0.03}{3 \times 10^{-3}}=10 .
$$

\subsection{Energy dissipation}

The energy dissipation in the system of SPH particles is further examined. As for incompressible Newtonian fluid with nominal dynamic viscosity $\mu_{0}$, the corresponding viscous dissipation (particularly referred to as "nominal viscous dissipation" hereafter) per unit volume, $\phi$, is defined by (in two dimensions)

$$
\phi=2 \mu_{0}\left[\left(\frac{\partial v^{x}}{\partial x}\right)^{2}+\left(\frac{\partial v^{y}}{\partial y}\right)^{2}\right]+\mu_{0}\left(\frac{\partial v^{y}}{\partial x}+\frac{\partial v^{x}}{\partial y}\right)^{2},
$$

where $v^{x}$ and $v^{y}$ are the $x$-component and $y$-component of the local velocity, respectively. Within the framework of SPH descriptions, the formulation of local viscous dissipation $\phi_{i}$ (based on particle $i$ ) would be

$$
\begin{aligned}
\phi_{i}= & \frac{\mu_{0}}{\rho_{i}^{2}}\left[2\left(\sum_{j} m_{j} v_{i j}^{x} \nabla_{i} W_{i j}^{x}\right)^{2}+2\left(\sum_{j} m_{j} v_{i j}^{y} \nabla_{i} W_{i j}^{y}\right)^{2}\right. \\
& \left.+\left(\sum_{j} m_{j} v_{i j}^{y} \nabla_{i} W_{i j}^{x}+\sum_{j} m_{j} v_{i j}^{x} \nabla_{i} W_{i j}^{y}\right)^{2}\right],
\end{aligned}
$$

where $v_{i j}^{x}$ and $v_{i j}^{y}$ are the relative velocities of particles $i$ and $j$ in the $x$ and $y$ directions, respectively. Also, $\nabla_{i} W_{i j}{ }^{x}$ and $\nabla_{i} W_{i j}^{y}$ denote the respective gradients of weighting function with respect to the location $\boldsymbol{r}_{i}$ in these two directions.

The nominal viscous dissipation of the whole system, $\phi_{\text {vis }}$, could be further obtained by the sum of local viscous dissipation as follows

$$
\phi_{\mathrm{vis}}=\sum_{i} \frac{m_{i}}{\rho_{i}} \phi_{i}
$$

Moreover, the steady-state total dissipation $\phi_{\text {total }}$ could be readily evaluated by means of energy balance:

$$
\phi_{\text {total }}=F V_{0} \text {, }
$$

where $F$ is the tangential force exerted on the moving boundary.

Figure 7 presents the variations of nominal viscous dissipation (of the whole system) and total dissipation with the ratio of viscous to elastic effect, which is obtained by changing the stiffness parameter with a constant driving velocity $V_{0}=2.5 \times 10^{-2}$. Note that the theoretical viscous dissipation $\left(4.793 \times 10^{-3}\right)$ of Newtonian fluid with nominal viscosity is also displayed in Figure 7 for comparison. It should be pointed that those data in Figure 7 are all based on time-averaged values due to the oscillation of velocity profile. When $v_{0} \dot{\gamma} / K$ is relatively small, the disparity between nominal viscous dissipation and total dissipation is quite remarkable, while both of them considerably deviate from the theoretical dissipation. As $v_{0} \dot{\gamma} / K$ further increases, nominal viscous dissipation and total dissipation seem decrease in a power-law fashion with strong nonlinear effect. Eventually, when $v_{0} \dot{\gamma} / K$ is sufficiently large, both of them become almost identical to the theoretical viscous dissipation. In nature, extremely large value of $v_{0} \dot{\gamma} / K$ indicates the exclusive dominance of viscous effect in the system, where the behavior of Newtonian fluid is well approximated with the minimum viscous dissipation in theory. On the other hand, the elastic effect would play an important role under comparatively small $v_{0} \dot{\gamma} / K$, resulting in

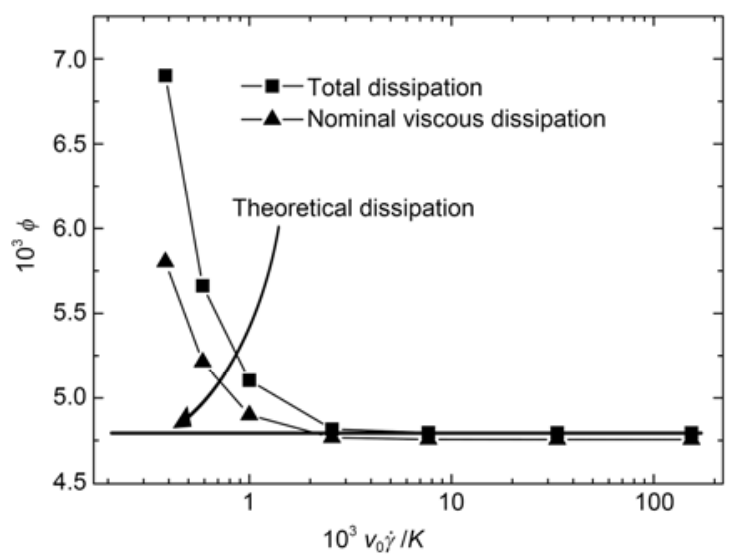

Figure 7 Variations of nominal viscous dissipation and total dissipation with the ratio of viscous to elastic effect. 
pronounced deviation from Newtonian fluid with much larger viscous dissipation. Nevertheless, the numerical simulation would become unstable for extremely large stiffness parameter, so that exclusive dominance of elastic effect is actually unattainable.

In fact, the inherent dissipation in the SPH simulation of inviscid Euler equation has been noted previously [40]. The disparity between nominal viscous dissipation and total dissipation in this study further demonstrates the presence of inherent dissipation in the SPH formulation of NavierStokes equation. To account for this disparity of energy dissipation, there should exist certain artificial viscosity in addition to the nominal viscosity, which has been revealed in the deviation of effective viscosity from nominal viscosity (Figure 5). Besides, the "velocity Verlet" integration algorithm is also performed, giving almost identical results. In summary, all these unphysical phenomena could be attributed to the discretization effect of SPH approach. More specifically, the fluid is only represented by quite finite number of particles, while the severe disorder of particle arrangement results in the low numerical accuracy. For a given particle number, this unavoidable numerical error is basically determined by the ratio of viscous to elastic effect.

\section{Conclusions}

By analyzing the physical behavior of SPH particles, a nondimensional number with the formulation $v_{0} \dot{\gamma} / K$ is proposed to characterize the relative dominance of viscous to elastic effect in the traditional SPH method. Through the simulation of two-dimensional Couette flow, it is found that the deviations of velocity profile, effective viscosity, and total dissipation from their theoretical counterparts all decrease as $v_{0} \dot{\gamma} / K$ increases. Therefore, this non-dimensional number could serve as a practical guide to evaluate the disparity between SPH simulation and corresponding Newtonian fluid. Due to the constraints from the Mach number and the ratio of viscous to elastic effect, there exists a critical Reynolds number below which the Newtonian behavior could be approximately obtained through suitable choice of model parameters.

Besides, as for the simulation of relatively high Reynolds number flows, extra corrections are needed to remedy the negative influence of elastic effect. Apart from those complicated and artificial modifications discussed previously, the improvement of SPH method from the angle of nondimensional number would be quite significant. Actually, when the non-dimensional number $v_{0} \dot{\gamma} / K$ is relatively small, the SPH particles essentially correspond to certain non-Newtonian fluid [30]. Of course, the underlying mechanism of this non-dimensional number is only preliminarily elucidated in this study, and our findings should also be further explored in various complex flows, which all call for future work.

This work was supported by the National Natural Science Foundation of China (21206167) and the Strategic Priority Research Program of the Chinese Academy of Sciences (XDA07080203). Also, the anonymous referees are gratefully acknowledged for their constructive comments.

1 Gingold R A, Monaghan J J. Smoothed particle hydrodynamics: Theory and application to non-spherical stars. Mon Not R Astron Soc, 1977, 181: 375-389

2 Lucy L B. A numerical approach to the testing of the fission hypothesis. Astron J, 1977, 82: 1013-1024

3 Monaghan J J. Simulating free surface flows with SPH. J Comput Phys, 1994, 110: 399-406

4 Shao S, Lo E Y M. Incompressible SPH method for simulating Newtonian and non-Newtonian flows with a free surface. Adv Water Resour, 2003, 26: 787-800

5 Zhou G, Ge W, Li B, et al. SPH simulation of selective withdrawal from microcavity. Microfluid Nanofluid, 2013, doi: 10.1007/s10404013-1165-1

6 Zhang M. Simulation of surface tension in 2D and 3D with smoothed particle hydrodynamics method. J Comput Phys, 2010, 229: 72387259

$7 \mathrm{Xu}$ X, Ouyang J, Jiang T, et al. Numerical simulation of 3D-unsteady viscoelastic free surface flows by improved smoothed particle hydrodynamics method. J Non-Newton Fluid Mech, 2012, 177-178: 109-120

$8 \mathrm{Xu} \mathrm{X}$, Ouyang J, Yang B, et al. SPH simulations of three-dimensional non-Newtonian free surface flows. Comput Methods Appl Mech Engrg, 2013, 256: 101-116

9 Shao S. Incompressible SPH simulation of wave breaking and overtopping with turbulence modeling. Int J Numer Methods Fluids, 2006, 50: 591-621

10 Violeau D, Issa R. Numerical modelling of complex turbulent freesurface flows with the SPH method: An overview. Int J Numer Method Fluid, 2007, 53: 277-304

11 Rafiee A, Thiagarajan K P. An SPH projection method for simulating fluid-hypoelastic structure interaction. Comput Method Appl Mech Engrg, 2009, 198: 2785-2795

12 Li S, Liu W K. Meshfree and particle methods and their applications. Appl Mech Rev, 2002, 55: 1-34

13 Belytschko T, Krongauz Y, Dolbow J, et al. On the completeness of meshfree particle methods. Int J Numer Method Eng, 1998, 43: 785-819

14 Liu M B, Liu G R. Restoring particle consistency in smoothed particle hydrodynamics. Appl Num Math, 2006, 56: 19-36

15 Koumoutsakos P. Multiscale flow simulations using particles. Annu Rev Fluid Mech, 2005, 37: 457-487

16 Liu W K, Jun S, Zhang Y F. Reproducing kernel particle methods. Int J Numer Method Fluid, 1995, 20: 1081-1106

17 Chen J K, Beraun J E, Carney T C. A corrective smoothed particle method for boundary value problems in heat conductions. Int $\mathrm{J} \mathrm{Nu}$ mer Method Eng, 1999, 46: 231-252

18 Liu M B, Xie W P, Liu G R. Modeling incompressible flows using a finite particle method. Appl Math Model, 2005, 29: 1252-1270

19 Jiang T, Ouyang J, Ren J, et al. A mixed corrected symmetric SPH (MC-SSPH) method for computational dynamic problems. Comp Phys Comm, 2012, 183: 50-62

20 Dilts G A. Moving-least-squares-particle hydrodynamics-I. Consistency and stability. Int J Numer Method Eng, 1999, 44: 1115-1155

21 Chaniotis A K, Poulikakos D, Koumoutsakos P. Remeshed smooth particle hydrodynamics for the simulation of viscous and heat conducting flows. J Comput Phys, 2002, 182: 67-90

22 Monaghan J J. Smoothed particle hydrodynamics. Rep Prog Phys, 2005, 68: 1703-1759

23 Posch H A, Hoover W G, Kum O. Steady-state shear flows via nonequilibrium molecular dynamics and smooth-particle applied 
mechanics. Phys Rev E, 1995, 52: 1711-1720

24 Hoover W G, Hess S. Equilibrium and nonequilibrium thermomechanics for an effective pair poterntial used in smooth particle applied mechanics. Physica A, 1996, 231: 425-438

25 Español P, Revenga M. Smoothed dissipative particle dynamics. Phys Rev E, 2003, 67: 026705

26 Tartakovsky A, Meakin P. Modeling of surface tension and contact angles with smoothed particle hydrodynamics. Phys Rev E, 2005, 72: 026301

27 Tartakovsky A M, Meakin P. Pore scale modeling of immiscible and miscible fluid flows using smoothed particle hydrodynamics. Adv Water Resour, 2006, 29: 1464-1478

28 Zhou G Z, Ge W, Li J H. A revised surface tension model for macro-scale particle methods. Powder Technol, 2008, 183: 21-26

29 Zhou G, Chen Z, Ge W, et al. SPH simulation of oil displacement in cavity-fracture structures. Chem Eng Sci, 2010, 65: 3363-3371

30 Zhou G Z, Ge W, Li J H. Smoothed particles as a non-Newtonian fluid: A case study in Couette flow. Chem Eng Sci, 2010, 65: 2258-2262

31 Morris J P, Fox P J, Zhu Y. Modeling low Reynolds number incompressible flows using SPH. J Comput Phys, 1997, 136: 214-226

32 Sigalotti L D G, Klapp J, Sira E, et al. SPH simulations of timedependent Poiseuille flow at low Reynolds numbers. J Comput Phys, 2003, 191: 622-638
33 Colagrossi A, Landrini M. Numerical simulation of interfacial flows by smoothed particle hydrodynamics. J Comput Phys, 2003, 191: 448-475

34 Hu X Y, Adams N A. A multi-phase SPH method for macroscopic and mesoscopic flows. J Comput Phys, 2006, 213: 844-861

35 Ellero M, Tanner R I. SPH simulations of transient viscoelastic flows at low Reynolds number. J Non-Newton Fluid Mech, 2005, 132: 61-72

36 Zhu H, Martys N S, Ferraris C, et al. A numerical study of the flow of Bingham-like fluids in two-dimensional vane and cylinder rheometers using a smoothed particle hydrodynamics (SPH) based method. J Non-Newton Fluid Mech, 2010, 165: 362-375

37 Zhu H, Zhou Z, Yang R, et al. Discrete particle simulation of particulate systems: Theoretical developments. Chem Eng Sci, 2007, 62: 3378-3396

38 Sun Q, Wang G, Hu K. Some open problems in granular matter mechanics. Prog Nat Sci, 2009, 19: 523-529

39 Grmela M, Öttinger H C. Dynamics and thermodynamics of complex fluids. I. Development of a general formalism. Phys Rev E, 1997, 56: 6620-6632

40 Hoover W G, Pierce T G, Hoover C G, et al. Molecular dynamics, smoothed-particle applied mechanics, and irreversibility. Comput Math Appl, 1994, 28: 155-174

Open Access This article is distributed under the terms of the Creative Commons Attribution License which permits any use, distribution, and reproduction in any medium, provided the original author(s) and source are credited. 